\title{
A NOTE ON THE SUM OF TWO CLOSED LATTICE IDEALS*
}

\author{
HEINRICH P. LOTZ ${ }^{1}$
}

\begin{abstract}
Suppose that $E$ is a locally convex lattice. The main results established in this note are: (a) If $I, J$ are $\sigma\left(E^{\prime}, E\right)$-closed lattice ideals in the dual $E^{\prime}$ of $E$, then $I+J$ is $\sigma\left(E^{\prime}, E\right)$-closed. (b) If $E$ is a Fréchet lattice (in particular, if $E$ is a Banach lattice) and if $I, J$ are closed lattice ideals in $E$, then $I+J$ is closed.
\end{abstract}

It is known that the sum of two closed lattice ideals in a Banach lattice is a closed lattice ideal (see Theorem 5.3 in [1] and Theorem 1.1 in [2]). In this note, we deal with the sum of two closed lattice ideals in a locally convex lattice and with the sum of the polars of two lattice ideals, that is, with the sum of two weak*-closed lattice ideals in the dual space.

A linear subspace $I$ of a vector lattice $E$ is a lattice ideal if $I$ is solid, that is, if $x \in I$ and $|y| \leqq|x|$ imply $y \in I$. The sum of two lattice ideals in a vector lattice is a lattice ideal. A closed linear subspace $I$ of a locally convex vector lattice $E$ is an ideal if and only if the polar $I^{\circ}$ of $I$ is a lattice ideal in the dual $E^{\prime}$ of $E$.

We refer the reader to [3] for further background information on locally convex vector lattices.

THEOREM 1. If $E$ is a locally convex vector lattice and if $I$ and $J$ are lattice ideals in $E$, then $(I \cap J)^{\circ}=I^{\circ}+J^{\circ}$.

Proof. It is clear that $(I \cap J)^{\circ} \supset I^{\circ}+J^{\circ}$. To prove the reverse inclusion, it would suffice to show that if $0 \leqq f \in(I \cap J)^{\circ}$, then $f \in I^{\circ}+J^{\circ}$ since $I^{\circ}+J^{\circ}$ is a lattice ideal in $E^{\prime}$. For $x \geqq 0$ in $E$, define

$$
\gamma(x)=\sup \{f(y): y \in[0, x] \cap I\} .
$$

Received by the editors October 26, 1972.

AMS (MOS) subject classifications (1969). Primary 4606.

Key words and phrases. Sum of closed lattice ideals.

${ }^{1}$ This research was supported by the National Science Foundation under Grant GP-28577.

* This article has not been proofread by the author because the Amer. Math. Soc. was unable to locate him. The address given at the end of the paper is the last address given by the author.

(c) American Mathematical Society 1974 
Then $\gamma$ is additive and positively homogeneous on the positive cone in $E$; consequently, $\gamma$ can be extended to a linear functional $g$ on $E$ (cf. proofs of $\mathrm{V}, 1.4$ and $\mathrm{V}, 1.6$ in [3]). Since $0 \leqq g \leqq f$ it follows that $g \in E^{\prime}$. Moreover, $f-g \in I^{\circ}$ and $g \in J^{\circ}$ since $[0, x] \cap I \subset I \cap J$ for each $x \in J$. Therefore, $f=(f-g)+g \in I^{\circ}+J^{\circ}$ which completes the proof.

REMARK. The linear functional $g$ constructed in the above proof is just the component of $f$ in $I^{\circ \perp}$ when $E^{\prime}$ is written as the order direct sum of the bands $I^{\circ}$ and $\left(I^{\circ}\right)^{\perp}$.

COROLlaRY. If $E$ is a locally convex vector lattice, then the sum of two $\sigma\left(E^{\prime}, E\right)$-closed lattice ideals in $E^{\prime}$ is $\sigma\left(E^{\prime}, E\right)$-closed. ${ }^{2}$

Proof. If $I$ and $J$ are $\sigma\left(E^{\prime}, E\right)$-closed lattice ideals in $E^{\prime}$, then the $\sigma\left(E^{\prime}, E\right)$-closure of $I+J$ is $\left(I^{\circ} \cap J^{\circ}\right)^{\circ}$; consequently, the conclusion follows immediately from Theorem 1 .

ThEOREM 2. Suppose that I and $J$ are lattice ideals in a locally convex vector lattice $E$. Then the mapping $(x, y) \rightarrow x+y$ is a weak homomorphism from $I \times J$ into $E$.

PRoof. It would suffice to show that the mapping $f \rightarrow\left(\left.f\right|_{I},\left.f\right|_{J}\right)$ (where $\left.f\right|_{I}$ denotes the restriction of $f$ to $\left.I\right)$ from $E$ into $I^{\prime} \times J^{\prime}$ has a $\sigma\left(I^{\prime} \times J^{\prime}, I \times J\right)$-closed range [3, IV 7.3]. This range is clearly contained in the $\sigma\left(I^{\prime} \times J^{\prime}, I \times J\right)$-closed subspace $G=\left\{(g, h): g \in I^{\prime}, h \in J^{\prime}, g(x)=h(x)\right.$ for all $x \in I \cap J\}$ of $I^{\prime} \times J^{\prime}$. If $(g, h) \in G$, then there exist $\hat{g}, \hat{h}$ in $E^{\prime}$ such that $\left.\hat{g}\right|_{I}=g,\left.\hat{h}\right|_{J}=h$ (by the Hahn-Banach theorem). Since $\hat{g}-\hat{h} \in(I \cap J)^{\circ}$ and since $(I \cap J)^{\circ}=I^{\circ}+J^{\circ}$ by Theorem 1 , it follows that $\hat{g}-\hat{h}=f_{1}+f_{2}$ where $f_{1} \in I^{\circ}, f_{2} \in J^{\circ}$. But then $(g, h)$ is the image of $f=\hat{g}-f_{1}=\hat{h}+f_{2}$ under the mapping $f \rightarrow\left(\left.f\right|_{I},\left.f\right|_{J}\right)$, that is, the range of this mapping is the $\sigma\left(I^{\prime} \times J^{\prime}, I \times J\right)$-closed subspace $G$.

\section{REFERENCES}

1. E. B. Davies, The structure and ideal theory of the predual of a Banach lattice, Trans. Amer. Math. Soc. 131 (1968), 544-555. MR 36 \#5654.

2. H. P. Lotz, Über das Spektrum positiver Operatoren, Math. Z. 108 (1968), 15-32. MR 39 \#1994.

3. H. H. Schaefer, Topological vector spaces, Macmillan, New York, 1966. MR 33 \#1689.

Department of Mathematics, University of Illinois, at Urbana-Champaign, URBANA, ILLINOIS 61801

${ }^{2}$ This Corollary was proved independently by S. Kaplan. 